\title{
Comunicación Gestual en la Infancia Temprana: Una Revisión de su Desarrollo, Relación con el Lenguaje e Implicancias de su Intervención
}

\section{Gestural Communication in Early Infancy: A Review of its Development, Relation With Language and Intervention Implications}

\author{
Chamarrita Farkas \\ Pontificia Universidad Católica de Chile
}

\begin{abstract}
El objetivo de este artículo es proveer una revisión actualizada y comprehensiva sobre el desarrollo y funciones de los gestos en la infancia, que apoye la labor de psicólogos, educadores y otros profesionales que investigan o trabajan con bebés y niños pequeños. Existe un creciente cuerpo de evidencias de que los gestos potencian y no obstaculizan el desarrollo del lenguaje. Gestos y lenguaje se desarrollan en forma paralela y comparten habilidades simbólicas subyacentes. Intervenciones educativas en bebés y sus familias fomentan el desarrollo intencionado de la comunicación gestual simbólica. Se revisan los resultados obtenidos en relación a aspectos del lenguaje, cognitivos y socio-afectivos. Se discuten sus implicancias generales y la necesidad de investigación adicional en aspectos específicos.
\end{abstract}

Palabras Clave: gestos, lenguaje, desarrollo, infancia.

\begin{abstract}
The aim of this article is to provide an actual and comprehensive overview of the development and functions of gesture during childhood, that could support the work of psychologists, educators, and other professionals conducting research or working with babies and toddlers. There is a growing body of evidence that gestures enhances, not hinders, language development. Gesture and language development parallels each other and share underlying symbolic abilities. Educational interventions in babies and their families encourage the intentional development of symbolic gestural communication. Results in relation with language, cognition, and socio-emotional aspects are presented. General implications and needs for additional research on specific aspects are discussed.
\end{abstract}

Keywords: gesture, language, development, infancy.

La comunicación constituye un proceso central a través del cual se intercambian y construyen significados con otros, a lo largo de todo el ciclo vital. Dentro de este proceso, el lenguaje gestual ocupa un importante lugar. Ya desde los primeros meses de vida los niños utilizan este lenguaje para manifestar sus necesidades, expresar sus sensaciones y sentimientos, y vincularse con otras personas así como con el mundo que los rodea (Acredolo \& Goodwyn, 1985).

El uso de gestos para comunicarse, las fases a través de las cuales éstos se desarrollan, y la relación específica de los gestos simbólicos con el lenguaje

Chamarrita Farkas, Escuela de Psicología, Pontificia Universidad Católica de Chile.

La correspondencia relativa a este artículo deberá ser enviada a la autora a: Escuela de Psicología, Pontificia Universidad Católica de Chile, Avda. Vicuña Mackenna 4860, Macul, Santiago, Chile. E-mail: chfarkas@uc.cl

La elaboración de este artículo contó con el financiamiento otorgado por el Fondo Nacional de Desarrollo Científico y Tecnológico (FONDECYT) a través del Proyecto $\mathrm{N}^{\mathrm{o}}$ 1060778 . así como con otros aspectos del desarrollo, ha sido ampliamente estudiada y documentada. Es así que el objetivo de este artículo es presentar la información que actualmente se dispone, abordando primero el desarrollo inicial de los gestos como una vía espontánea de comunicación y su relación con el lenguaje, para luego revisar los resultados que han conseguido programas de intervención cuyo objetivo ha sido desarrollar de manera intencional este tipo de comunicación en infantes y niños pequeños.

\section{Estudios Sobre el Desarrollo Espontáneo del Lenguaje Gestual}

Para revisar el desarrollo de los gestos intencionales tempranos, se considerará la clasificación de gestos deícticos y gestos simbólicos propuesta por Capirci, Iverson, Pizzuto y Volterra (1996), y a la cual se adhieren la mayoría de las investigaciones actuales (Camaioni, Aureli, Bellagamba \& Fogel, 2003; Rodrigo, González, Ato, Rodríguez \& de Vega, 2006), para finalmente revisar brevemente los gestos icónicos. 


\section{Gestos Deícticos}

Los gestos deícticos aparecen entre los 9 y 12 meses de edad. Se refieren a apuntar, mostrar, ofrecer, dar y realizar peticiones en forma de ritual (e. g. extender el brazo con la mano abierta y la palma hacia arriba) (Bates, Benigni, Bretherton, Camaioni \& Volterra, 1979). Ya Piaget (1965) proponía que alrededor de los 10 meses de edad aparecían los "gestos de ejecución", los cuales se referían a acciones como el observar insistentemente un objeto para indicar que se le quiere, dirigir la atención del adulto hacia un objeto dándoselo, o apuntar objetos para demostrar interés o necesidad. Estos gestos se corresponden a la vez con los gestos protodeclarativos y protoimperativos descritos por Premack y Woodruff (1978), en los cuales los gestos se utilizan para hacer peticiones (protoimperativos) o para solamente mostrarle un objeto a otras personas (protodeclarativos). Otros autores optan por subdividirlos en apuntar, los cuales aluden a señalar a una persona u objeto, y gestos instrumentales, los cuales consideran el pedir, mostrar o dar algo (Rodrigo et al., 2006).

Estos gestos intencionales tempranos constituyen un claro avance sobre el actuar meramente sobre los objetos, pero todavía son primitivos en cuanto a la sofistificación de la representación, ya que el significado del referente es claro sólo si el observador sigue la trayectoria del gesto hacia su objetivo. Pero constituyen un importante paso en el desarrollo simbólico y pavimentan el camino al aprendizaje del lenguaje verbal (Goodwyn, Acredolo \& Brown, 2000).

El desarrollo de los gestos deícticos requiere de una intención comunicativa triádica, la cual se refleja en los episodios de atención compartida, sin la cual éstos no son posibles (Rodrigo et al., 2006). Estudios de seguimiento del desarrollo de estos gestos hasta los 2 años de edad indican que los gestos deícticos emergen de igual manera en niños y en niñas (Crais, Day \& Cox, 2004), se incrementan linealmente y se correlacionan positivamente con el desarrollo del lenguaje y del discurso (Camaioni et al., 2003).

\section{Gestos Simbólicos o Representacionales}

Los gestos simbólicos surgen entre los 12 y 15 meses de edad, y constituyen acciones físicas simples que pueden ser usadas para representar objetos y eventos, así como expresar deseos, necesidades, pensamientos y emociones. Estos gestos tienen una función comunicativa y nominativa, representan un referente específico, conllevan su significado en su forma y éste no cambia con el contexto, sustituyen el habla y tienen una función utilitaria hasta que los equivalentes verbales sean posibles (Acredolo \& Goodwyn, 1988, 1990; Goodwyn et al., 2000; Iverson, Capirci \& Caselli, 1994).

Algunos autores subdividen estos gestos en gestos sociales, los cuales se insertan en una rutina social (e.g. mover la mano para saludar o despedirse), y gestos simbólicos o inactivos, los cuales representan la forma o función de un referente (e.g. brazos extendidos a los lados del cuerpo, moviéndolos hacia arriba y hacia abajo, refiriéndose a un pájaro) (Rodrigo et al., 2006).

Estos gestos se desarrollan de manera diferente a los gestos deícticos; estudios de seguimiento en niños muestran que a los 20 meses de edad los gestos simbólicos declinan en relación al incremento de la producción verbal (Iverson et al., 1994) lo cual sustentaría la hipótesis que estos gestos sólo cumplen una función utilitaria, mientras los equivalentes verbales sean posibles, hipótesis que será luego revisada a la luz de los resultados en desarrollo intencional de estos gestos en infantes y preescolares.

La comunicación gestual combina cuatro elementos o componentes fonológicos: la forma o configuración de la mano (u otra parte del cuerpo), el lugar en que el signo se forma, el movimiento que se hace y su orientación (Daniels, 2001). Se le considera como un lenguaje propiamente tal, y es similar al lenguaje verbal en cuanto al uso de gestos arbitrarios, a los facilitadores del aprendizaje, a la forma en que se inicia y desarrolla, y por el tipo de errores que se comete (Daniels, 2001; Mehler \& Dupoux, 1994).

El desarrollo de estos gestos se da en una variedad impresionante, lo cual es atribuido a que los adultos le modelan al niño no sólo los gestos en sí mismos, sino el uso de éstos como herramientas para la comunicación. Así, el niño aprende tanto la forma como la función (Goodwyn et al., 2000). La importancia de estos gestos para el desarrollo del lenguaje ya no es un tema de discusión. Contribuyen en el desarrollo, tanto de un modo indirecto comunicando aspectos no hablados del estado cognitivo del niño, como de una manera directa en la cual ofrecen al niño una manera más simple de expresar y explorar ideas que serían difíciles de pensar en un formato verbal (Goldin-Meadow, 2000). Le permiten al niño explorar más allá de los confines del lenguaje que está hablando y nos proveen de la oportunidad única de observar las habilidades del niño como "constructor" del lenguaje (Goldin-Meadow, 2002). 


\section{Gestos Icónicos}

Una vez que el niño ya ha adquirido el lenguaje verbal, los gestos simbólicos o representacionales evolucionan hacia los gestos icónicos, descritos por Nicoladis, Mayberry y Genesee (1999). Estos aparecen aproximadamente a los 3 años de edad, cumplen una función afirmativa y acompañan al habla, en vez de sustituirla. Alrededor de los 3 años de edad hay una fuerte tendencia del niño a depender de una parte de su cuerpo para representar, por ejemplo, una herramienta (e.g. usa su dedo como si fuera el cepillo de dientes), pero hacia los 5 años la capacidad representacional del niño se ha complejizado hasta el punto que es capaz de entender o producir la acción relevante por sí misma, como si la herramienta estuviera allí (e.g. mover la mano como si estuviera sosteniendo un cepillo de dientes). Por tanto, a esta edad el símbolo se ha distanciado del referente lo suficiente como para no necesitar aparentemente ningún símbolo concreto de la herramienta (Boyatzis \& Watson, 1993; O’Reilly, 1995), lo cual se corresponde con el proceso de decontextualización, el cual es común a las modalidades gestual y vocal (Bates et al., 1979; Iverson et al., 1994).

\section{Relación Entre Gestos y Desarrollo del Lenguaje}

Tanto la literatura como las investigaciones han revisado la relación entre el desarrollo de los símbolos verbales y los gestuales en las distintas etapas del ciclo vital del niño. El desarrollo de gestos de manera espontánea en el bebé ocurre de manera natural, ligado al ciclo vital, apreciándose que suelen ser bastante comunes entre los 10 y 24 meses de edad, y se aprenden a través de rutinas interactivas con los adultos, de la observación de modelos o prestarse de las acciones realizadas con el objeto de referencia (Acredolo \& Goodwyn, 1985, 1988; Folven \& Bonvillian, 1991).

Este aprendizaje de los gestos se da en un momento en que en el lactante aparece una necesidad de comunicarse, acompañada de un gran desarrollo cognitivo, y donde la habilidad gestual aparece más tempranamente al desarrollo de la habilidad para producir palabras (Acredolo \& Goodwyn, 2001; Goodwyn \& Acredolo, 1993).

\section{Los Gestos Predicen los Posteriores Hitos del Lenguaje}

Los gestos son concebidos como indicadores relevantes de una etapa prelingüistica, como conductas que preceden y preparan la emergencia del lenguaje verbal (Volterra, Caselli, Capirci \& Pizzuto, 2005). Así, podemos plantear que los gestos durante los dos primeros años de vida predicen los posteriores hitos del lenguaje. Capirci et al. (1996) encontraron que gestos únicos así como combinaciones gesto-palabra producidos a los 16 meses, se correlacionaban significativamente con la producción vocal total medida a los 20 meses de edad. Especialmente significativa resultaba además la correlación entre las combinaciones de gestos deícticos o representacionales con palabras a los 16 meses, y la producción vocal total a los 20 meses. Estos resultados son corroborados por McEachern y Haynes (2004), quienes constataron que la combinación gesto-palabra correspondía a una etapa de transición entre la etapa de una palabra y la etapa de dos o más palabras.

A su vez, un estudio realizó un seguimiento a 10 niños que se encontraban en el $10 \%$ más bajo de producción de lenguaje verbal para su edad desde los 18 a los 29 meses de edad, y comprobó que los niños que tenían un retraso real en el aprendizaje del habla tenían a su vez un desempeño significativamente más pobre en las tareas gestuales, en comparación a niños normales (Thal, Tobias \& Morrison, 1991).

\section{Los Gestos y el Lenguaje Revelan Operaciones Cognitivas}

La secuencia que siguen los gestos desde los gestos deícticos a los icónicos, pasando por los simbólicos o representacionales, revela el distanciamiento gradual del self en relación a los objetos, que subyace al desarrollo simbólico, alcanzando en cada etapa un mayor distanciamiento en relación al referente (Capone \& McGregor, 2004).

El desarrollo de gestos y palabras está altamente conectado tanto desde el punto de vista evolutivo y neurológico. Se da una sobreposición en el control neuronal del discurso y de los gestos de modo que se extiende una activación neuronal de una zona cerebral a otra, que puede explicar su co-ocurrencia (Capone $\&$ McGregor, 2004). Estudios indican que los gestos simbólicos refuerzan la palabra, mientras que la emisión de la palabra inhibe la producción del gesto. Ello pareciera indicar que palabras y gestos son codificados como una señal individual por un sistema único de comunicación (Bernardis \& Gentilucci, 2006). 
Estudios realizados en niños de distintas culturas (norteamericanos e italianos), expuestos a distintas modalidades de lenguaje (hablado y de señas) y que manifiestan tanto un desarrollo normal como alterado (Síndrome de Down y Síndrome de Willia$\mathrm{ms}$ ) concluyen una continuidad entre el desarrollo prelingüístico y lingüístico, donde las habilidades gestuales simbólicas y las producciones vocales están totalmente conectadas entre sí y evolucionan en conjunto a otras habilidades cognitivas generales (Volterra et al., 2005).

\section{Los Gestos Facilitan el Desarrollo del Lenguaje}

El inicio de la ejecución de los gestos varía ampliamente de niño en niño, lo cual se relaciona con una serie de aspectos como el interés del niño por comunicarse, el desarrollo de destrezas cognitivas relacionadas (memoria, imitación, atención) y la frecuencia con que los padres modelan estos gestos (Acredolo \& Goodwyn, 2001; García, 1999; Moore). Se ha observado a través de estudios empíricos que los padres entregan a los niños tanto rótulos verbales como rutinas gestuales durante los mismos contextos de atención compartida, y que mientras más gestos producen los padres, más gestos realizan los niños (Namy, Acredolo \& Goodwyn, 2000). Así, las primeras evidencias del inicio de esta comunicación intencional prelingüística se da a través de conductas de atención conjunta, en las cuales el niño antes, durante o después de la realización del gesto, hace contacto ocular con el adulto (Gómez, Sarriá \& Tamarit, 1993).

A la vez, se ha observado una alta correlación entre la producción de gestos de la madre y la producción de gestos y palabras del niño, así como el tamaño de su vocabulario. Esta correlación se mantiene estable a través del tiempo, e incluso se alude a una especie de "maternaje gestual". Esto ha sido constatado en estudios de niños italianos de 16 y 20 meses de edad y sus madres (Iverson, Capirci, Longobardi \& Caselli, 1999) así como en niños españoles de 2 y 3 años (Rodrigo et al., 2006), lo cual indica que esta articulación entre la producción de gestos de la madre y la de su hijo se da en diferentes culturas.

Por otra parte, cuando los padres son estimulados a modelar gestos al niño en interacciones cotidianas, el uso de gestos simbólicos comienza más temprano (Goodwyn \& Acredolo, 1993), los niños demuestran

\footnotetext{
1 “Gestural Motherese" (Iverson et al., 1999).
}

un mayor uso de estos gestos que lo observado en el desarrollo espontáneo de los mismos (Goodwyn et al., 2000) y adelantan significativamente el inicio del lenguaje (Acredolo \& Goodwyn, 1990; Goodwyn et al., 2000).

Estudios indican la relación entre los gestos simbólicos que los niños realizan espontáneamente y el lenguaje. Por ejemplo, en un estudio longitudinal con 16 niños, desde los 11 a 20 meses de edad, se vio una correlación inversa significativa $(r=-.48, p<$ 0.01 ) entre la cantidad de gestos relativos a objetos y la edad del niño, en el hito de las 10 palabras. En otras palabras, cuando más gestos realizaba, más tempranamente el niño alcanzaba el hito de 10 palabras en su vocabulario (Thal \& Bates, 1988).

\section{Los Gestos Cumplen una Función en la Comunicación, que va más Allá del Destinatario de la Misma}

Estudios empíricos realizados en niños ciegos de nacimiento muestran que estos realizan gestos simbólicos al comunicarse, que estos gestos se dan en la mayoría de los contextos en los cuales lo hacen los niños normales y que los gestos que realizan se parecen tanto en forma como en contenido a los de los niños que pueden ver. Estos datos indican que los gestos cumplen una función importante para la persona que habla, independientemente de su impacto en la persona que escucha, la cual tiene que ver con la forma en que se representa el abordaje de una situación. Los niños ciegos y videntes no se diferencian en su habilidad de producir gestos per se, sino en la forma predominante en que ellos se representan las situaciones (Iverson \& Goldin-Meadow, 1997). Tal como Goldin-Meadow y Morford (1985) manifiestan, "la comunicación en los seres humanos es un fenómeno resiliente; cuando se impide que salga por la boca, emana de manera casi incontenible por los dedos" (p. 146).

\section{La Relación Gesto-Palabra Varía con la Edad}

Esta habilidad general inicial de aprender y producir gestos como referencia simbólica y su relación con las palabras, va variando de acuerdo a la edad del niño. Además, los gestos simbólicos complementan las formas tempranas del habla (Acredolo \& Goodwyn, 1988; Iverson et al., 1994) y muestran una función transicional en el segundo año de vida (Capirci et al., 1996). En un estudio realizado con 48 niños sobre la categorización de objetos desconocidos, se pudo apreciar que a los 18 meses de edad 
los niños interpretaban espontáneamente el gesto, al igual que la palabra, como el nombre de un objeto, en cambio a los 26 meses lo hacían sólo con la palabra (Namy \& Waxman, 1998).

Entre los 12 y 18 meses de edad, los gestos y el habla son en general mutuamente excluyentes, siendo los gestos eventualmente reemplazados por palabras, pero no en forma inversa (Capirci et al., 1996). En una investigación, la mayoría de los niños evaluados a los 16 meses tenían un mayor vocabulario gestual que verbal y preferían comunicarse en la modalidad gestual, mientras que a los 20 meses tenían un mayor vocabulario verbal que gestual y empleaban las palabras de manera más frecuente que los gestos (Iverson et al., 1994). Estos resultados indican que la habilidad general de aprender símbolos se desarrolla en una tendencia más focalizada al uso de palabras a través del desarrollo, como la forma simbólica predominante. El desarrollo de los gestos ayuda a responder a la demanda de desarrollar habilidades simbólicas y ayuda al niño en la transición a relaciones palabra-referente más abstractas.

\section{La Relación Gestos-Palabras se Mantiene a lo Largo del Desarrollo}

En la etapa preescolar, los gestos se encuentran estrechamente relacionados con el habla. Nicoladis et al. (1999) encontraron que niños de 24-42 meses de edad usaban típicamente gestos junto con palabras más que gestos solos, y que los intercambios comunicativos eran de mayor duración cuando se acompañaban de gestos icónicos que cuando lo hacían con gestos deícticos o con ausencia de gestos. Además de la asociación entre el uso de gestos y los avances en el lenguaje expresivo, también investigaciones han encontrado que la asociación entre gestos y vocabulario comprensivo se mantiene en los años preescolares (O’Reilly, Painter \& Bornstein, 1997).

En los años escolares, el uso de gestos continúa apoyando la expresión de conceptos más abstractos, además de conducir a la adquisición conceptual por sí mismos. Goldin-Meadow y sus colegas tienen una basta línea de investigación que muestra el uso de los gestos para acceder al pensamiento formal en niños a partir de 5 años. Cuando se le pide a niños escolares en los primeros años, que expliquen conceptos como la conservación de Piaget o la equivalencia matemática, usan en sus explicaciones tanto gestos espontáneos como formas verbales (Capone \& McGregor, 2004; Goldin-Meadow, 2000, 2002).

\section{Gestos Espontáneos Versus Desarrollo Intencional de la Comunicación Gestual Simbólica: Programas de Intervención en Comunicación Gestual}

Como ya fue mencionado, el niño puede desarrollar los gestos simbólicos a través de la imitación de los adultos y en especial de sus figuras significativas, o crearlos de manera espontánea. Esta habilidad para crear espontáneamente sus propios gestos simbólicos como una forma de evadir los obstáculos que implican las demandas articulatorias del lenguaje verbal, ha sido observada tanto en estudios de casos, como en estudios transversales y longitudinales (Acredolo \& Goodwyn, 1985, 1988).

Desde la perspectiva que estos gestos permiten establecer un puente de comunicación con el niño hasta la aparición del habla, y que incluso cuando ésta aparece no todas las palabras tienen el mismo nivel de dificultad y por tanto el gesto puede ayudar hasta que la palabra pueda ser dicha correctamente o comprendida (Acredolo \& Goodwyn, 2001), e incluso estudios en preescolares de 3 a 5 años de edad que señalan que estos gestos continúan jugando un rol importante en la comprensión del lenguaje en la medida que los niños desarrollan habilidades lingüísticas más complejas (Kelly, 2001), nace la iniciativa de desarrollar de manera intencionada estos gestos en lactantes sin déficit sensorial.

Las intervenciones cuyo objetivo es desarrollar intencionadamente la comunicación gestual entre el niño normal y sus padres, surgen en Estados Unidos en los años noventa con las psicólogas Linda Acredolo y Susan Goodwyn, quienes fomentan el desarrollo de gestos sencillos y fáciles de aprender, con una fuerte vinculación con la forma o función del referente, posibles de ser modificados de acuerdo a la cultura familiar y con un énfasis en los aspectos emocionales a la base de la comunicación. La intervención propuesta por dichas psicólogas (BABY SIGNS $\AA$ ) se ha ido difundiendo a casi todos los estados de Estados Unidos así como en otros países como Canadá, Korea, Hong Kong e India, y está avalada por una serie de investigaciones y publicaciones.

También en la década de los 90 surge la propuesta del educador Joseph García quien promovió estas intervenciones, pero con un énfasis en el desarrollo cognitivo y del lenguaje. García proponía por tanto enseñarles a los niños desde temprana edad el lenguaje de señas americano usado por las personas sordas (ASL), con el objetivo de no sólo estimular el 
desarrollo intencionado de la comunicación gestual, sino enseñarle al niño un "segundo idioma". Este autor está retirado en la actualidad, pero su programa SIGN with your BABY ${ }^{\circledR}$ sigue difundiéndose en Estados Unidos, Canadá e Inglaterra. En esta misma línea se encuentran las investigaciones de Marilyn Daniels las cuales parten con el estudio de los gestos en niños sordos, para luego abordar su estudio en niños sin déficit auditivo (para mayor información, ver su libro Dancing with words: Signing for hearing children's literacy, 2001).

En un estudio descriptivo de la comunicación gestual espontánea, se describió la presencia de un promedio de 5 gestos en los niños observados, en los primeros dos años de vida (Acredolo \& Goodwyn, 1988), mientras que al desarrollarse de manera intencional la comunicación gestual en los niños, éstos alcanzaban un promedio de 20.38 gestos $(S D=12.64)$. Ello constituye una evidencia que el desarrollo intencionado de comunicación gestual en los niños es exitosa.

Una serie de investigaciones se han realizado considerando los efectos del desarrollo intencionado de la comunicación gestual en bebés, en cuanto al desarrollo del lenguaje, desarrollo cognitivo y desarrollo socio-emocional, cuyos principales resultados se presentan a continuación.

\section{Comunicación Gestual y Lenguaje Verbal}

Diversas investigaciones han constatado la relación entre comunicación gestual y desarrollo del lenguaje verbal. Estudios de tipo correlacional muestran altas correlaciones entre la cantidad de gestos simbólicos utilizados y tamaño de vocabulario verbal, a los 19 y 24 meses de edad (Acredolo \& Goodwyn, 1988; Goodwyn, 1986). Luego, en un estudio piloto realizado con 6 niños cuyos padres fueron motivados a comunicarse con ellos con gestos simbólicos, se constató que a los 24 meses de edad su desarrollo verbal era mejor en comparación a las normas nacionales (Acredolo \& Goodwyn, 1990).

En estudios comparativos se observó que los niños que habían sido estimulados a comunicarse gestualmente con sus figuras significativas desde temprana edad, eran capaces de entender más palabras, su vocabulario era mayor, y se involucraban en juegos más sofisticados, en comparación a los niños del grupo control (Goodwyn \& Acredolo, 1993, 1998).

En un estudio longitudinal se investigó a 103 niños desde los 11 hasta los 36 meses de edad, para lo cual se les aplicó el MacArthur Communicative
Development Inventory (CDI), Sequenced Inventory of Communicative Development (SICD) y Receptive- and Expressive-One-Word-Picture-Vocabulary Tests (ROWPVT \& EOWPVT) en diferentes edades. En dicho estudio se comprobó que los niños del grupo de estudio lograban de manera significativa un mejor desarrollo en su lenguaje expresivo y comprensivo, en comparación a los niños del grupo control en todas las pruebas aplicadas, y en las distintas edades evaluadas. Lo mismo ocurrió en tareas de discriminación fonética y desarrollo de la sintaxis a los 24 meses (Goodwyn et al., 2000).

La influencia del desarrollo intencional de la comunicación gestual temprana en el lenguaje, permanece a través del tiempo. En un estudio longitudinal realizado con 34 niños desde los 2 a los 4 años de edad, se encontraron asociaciones concurrentes significativas entre lenguaje y gestos (O’Reilly et al., 1997).

\section{Comunicación Gestual y Desarrollo Cognitivo}

Un hito importante dentro del desarrollo cognitivo, es el logro de episodios de atención compartida, la cual de acuerdo a Baron-Cohen (1994) ocurre alrededor de los 10-12 meses de edad y constituye la evidencia del logro de representaciones secundarias en el niño y por tanto de la capacidad de simbolizar y "hacer como si". Varios estudios describen la vinculación entre la comunicación gestual y las conductas de atención conjunta (Gómez et al., 1993; Tomasello \& Farrar, 1986).

En un estudio realizado con 103 niños, parte de ellos participaron a los 11 meses de edad en un Programa de Comunicación Gestual. Al evaluar a los 19 y 24 meses de edad como ellos iniciaban episodios de atención conjunta con sus madres en interacciones de juego, se constató que los niños del grupo de estudio iniciaban episodios de atención compartida con sus madres en una frecuencia significativamente mayor en comparación a los del grupo control, y que utilizaban estrategias significativamente más complejas para este fin (Moore et al., 2001). A la vez, los niños que alcanzaban los puntajes más altos en el inicio de estos episodios, exhibían habilidades más avanzadas de lenguaje expresivo, lo cual apoya la relación entre estos episodios y el desarrollo del lenguaje, ya sea verbal o gestual.

También ha sido estudiada la relación entre el desarrollo intencionado de gestos simbólicos y capacidad cognitiva. En un estudio longitudinal con 43 niños, de los cuales una parte aprendió 
a comunicarse a través de gestos con sus padres cuando eran bebés, se comprobó que al evaluar su desarrollo cognitivo a través del WISC-III al término del 2 do año básico, los niños del grupo de estudio que habían aprendido a comunicarse con gestos $(n=$ 24) lograban puntajes significativamente más altos en las escalas verbal $(F[1,41]=9.45, p=0.0038)$, motora $(F[1,41]=6.19, p=0.017)$, y escala total $(F[1,41]=12.06, p=0.0012)$. De estas escalas, la diferencia mayor se daba en la escala verbal (CI 116 versus 103), seguida de la escala total (CI 114 versus 102). Finalmente, aunque la diferencia en la escala motora era menor (CI 109 versus 101), igualmente resultó ser significativa (Acredolo \& Goodwyn, 2000).

\section{Comunicación Gestual y Desarrollo Socio- Emocional}

En observaciones realizadas así como a través del reporte de los padres, se ha visto que los niños que han sido estimulados en la utilización de los gestos para comunicarse, se frustran y enojan menos, lloran menos, y no utilizan preferentemente los gritos como formas de llamar la atención (Acredolo \& Goodwyn, 2001). Ello puede entenderse considerando que la posibilidad de comunicarse a través de gestos le permite al niño transmitir de una manera sencilla y rápida sus necesidades, lo cual disminuye su frustración y le resulta tremendamente reforzante, en la medida que el otro comprende lo que él trata de transmitirle.

Investigaciones han constatado que los niños pequeños pueden representarse conceptos relativos a emociones y sentimientos de manera simbólica, y pueden articularlos explícitamente en los intercambios comunicativos con otros. En un estudio realizado por Vallotton (en prensa) con 22 niños, se pudo detectar la presencia de gestos relativos a feliz, pena o asustado, a partir de los 10.9 meses de edad, y que generalmente los niños preferían iniciar ellos los gestos simbólicos más que imitar al adulto. Lo anterior nos presenta la capacidad del niño de representarse y comunicarle a otros estados emocionales en la etapa preverbal, y nos abre una ventana a sus mentes infantiles.

La capacidad del niño de poderle comunicar al adulto sus necesidades y emociones influirá además en la interacción que establecerá con éste. En un estudio realizado con 29 niños y sus padres, se comprobó que los padres que habían participado de un programa de comunicación gestual $(n=16)$, presentaban una disminución del estrés asociado a los aspectos de sentirse reforzados por sus hijos y en aceptar sus conductas (medido con el PSI), lo cual incidía en menor estrés asociado al rol parental, y por tanto mejor vinculación con sus hijos (Vallotton, 2005). En este mismo estudio se observó además en los padres un incremento de la focalización de la atención en su hijo y un mayor nivel de sintonía afectiva padres-bebé. Los niños del grupo estudio por su parte presentaron una mayor frecuencia de claves sociales y out put verbales durante las interacciones y menores indicadores de estrés, en comparación a los niños del grupo control.

\section{Conclusiones}

A través de esta revisión, se puede concluir que la evidencia empírica apoya contundentemente el que los gestos refuerzan en vez de obstaculizar, el desarrollo del lenguaje. El desarrollo de los gestos, tanto deícticos como simbólicos, se da en un momento en que en el lactante aparece una necesidad de comunicarse, acompañada de un gran desarrollo cognitivo, pero en el cual la madurez fonorrespiratoria todavía no permite el surgimiento del habla. Incluso cuando ésta aparece, se da de manera gradual y con distintos niveles de dificultad para cada palabra (Acredolo \& Goodwyn, 2001; Goodwyn \& Acredolo, 1993). Los gestos cumplen un importante rol en esta etapa, precediendo, facilitando y complementando el desarrollo de los hitos del lenguaje.

Los gestos cumplen una función en el proceso comunicativo, que va más allá de las personas a las cuales está destinado. Comparten a la vez un proceso de desarrollo similar al lenguaje, existiendo evidencia que apoya el que palabras y gestos son codificados como una señal individual por un sistema único de comunicación (Bernardis \& Gentilucci, 2006). Tanto en la comunicación gestual como verbal subyacen similares funciones simbólicas y cognitivas, y aunque la relación palabra-gesto varía según la edad, ésta se mantiene a lo largo de todo el ciclo vital.

La evidencia revisada nos abre una importante vía de estimulación del lenguaje en lactantes y niños pequeños, la cual podría considerar e incluir la modalidad gestual además de la verbal, en las interacciones que establecen los padres, educadores y otras figuras significativas en la vida del niño, especialmente cuando la modalidad verbal no está completamente desarrollada. Los padres suelen reportar pataletas y conductas disruptivas que se 
asocian con retraso o trastornos en el desarrollo del lenguaje, y con la frustración resultante de la imposibilidad de comunicarse. El uso de los gestos puede permitir al niño comunicar sus deseos y necesidades, reduciendo el estrés y frustración tanto de sí mismo como de sus padres (Acredolo \& Goodwyn, 2001; Capone \& McGregor, 2004).

Como ha sido señalado, el lenguaje gestual se desarrolla de manera espontánea, dentro del proceso de adquisición de la comunicación, y se mantiene durante todo el ciclo vital. Pese a ello no se le da la relevancia que requiere, y el foco de padres y educadores está puesto exclusivamente en el desarrollo del lenguaje verbal, tanto en sus formas orales como escritas. La evidencia señala por tanto que el uso de gestos simbólicos decrece en relación a las palabras, durante el crecimiento del niño (Iverson et al., 1994) pese a que su relevancia en fases posteriores del desarrollo cognitivo y del lenguaje ha sido demostrada (Acredolo \& Goodwyn, 2000; Goldin-Meadow, 2000, 2002; Goodwyn et al., 2000; Volterra et al., 2005). Programas que ayuden a padres y otros cuidadores a estar informados y entrenados para reconocer, aceptar y fomentar estas expresiones gestuales como comunicación plantean sus ventajas, considerando que los gestos pueden actuar como agentes indirectos del desarrollo. Una comunicación exitosa e interacciones familiares positivas no sólo contribuyen al desarrollo de las habilidades lingüísticas del niño, sino en la construcción de su autoestima (Capone \& McGregor, 2004).

A la vez, en niños mayores, los gestos continúan acompañando el discurso del niño ya que para éste, puede ser más fácil o posible expresar una idea o concepto a través de la expresión gestual y no hablada. El estar atentos a ello nos permite detectarla y "ponerla en palabras", lo cual puede resultar de suma utilidad en ambientes tanto educativos como terapéuticos (Capone \& McGregor, 2004).

Lo anterior ha llevado a generar programas que desarrollan intencionadamente los gestos simbólicos en lactantes, a través de la interacción con padres y otros cuidadores. Estas intervenciones han demostrado ser exitosas, avalando sus beneficios en el desarrollo cognitivo y del lenguaje, beneficios que se mantienen en el tiempo (Acredolo \& Goodwyn, 1988, 1990, 2000; Gómez et al., 1993; Goodwyn, 1986; Goodwyn \& Acredolo, 1993, 1998; Goodwyn et al., 2000; Moore et al., 2001, O'Reilly et al., 1997). A la vez, existe evidencia que no ha sido revisada en profundidad en este artículo, pero que avala los beneficios de la comunicación gestual en niños con alteraciones en su lenguaje ("habladores tardíos", niños en riesgo físico o social, déficit específicos del lenguaje, Síndrome de Down, Síndrome de Williams, autismo). Esta es una de las áreas más fructíferas para futuras investigaciones en intervención (Capone \& McGregor, 2004).

También se ha documentado los beneficios de la estimulación de la comunicación gestual temprana en el desarrollo socio-emocional de los niños, permitiéndoles expresar emociones, disminuyendo la frustración, y mejorando las interacciones padresbebé (Acredolo \& Goodwyn, 2001; Vallotton, en prensa; Vallotton, 2005). Nuevas y más acuciosas investigaciones deben ser realizadas, considerando la influencia de estas intervenciones en aspectos tales como el desarrollo de la autorregulación emocional en el niño en el primer año de vida, su efecto en las interacciones tempranas con padres y otros cuidadores, y la construcción de vínculos sanos. En el preescolar a la vez debiera estudiarse su influencia en la adquisición y desarrollo de habilidades sociales.

Finalmente, otros campos de investigación futura debieran considerar el impacto de los gestos simbólicos en distintas culturas y en distintas poblaciones de niños con alteraciones de lenguaje, la eficacia de los gestos como parte de programas para potenciar el desarrollo del lenguaje y la validación de los mismos como indicadores del desarrollo cognitivo y lingüístico.

\section{Referencias}

Acredolo, L. \& Goodwyn, S. (1985). Symbolic gesturing in language development: A case study. Human Development, $28,40-49$.

Acredolo, L. \& Goodwyn, S. (1988). Symbolic gesturing in normal infants. Child Development, 59, 450-499.

Acredolo, L. \& Goodwyn, S. (1990). Sign language in babies: The significance of symbolic gesturing for understanding language development. En R. Vasta (Ed.), Annals of Child Development, Vol. 7 (pp. 1-42). London: Jessica Kingsley Publishers.

Acredolo, L. \& Goodwyn, S. (2000, julio). The long-term impact of symbolic gesturing during infancy on IQ at age 8 . Ponencia presentada en Meetings of the International Society for Infant Studies, Brighton, UK.

Acredolo, L. \& Goodwyn, S. (2001). Los gestos del bebé. Barcelona: Oniro.

Baron-Cohen, S. (1994). How to build a baby that can read minds: Cognitive mechanisms in mindreading. Current Psychology of Cognition, 13(5), 513-552.

Bates, E., Benigni, L., Bretherton, J., Camaioni, L. \& Volterra, V. (1979). The emergence of symbols: Cognition and communication in infancy. New York: Academic Press.

Bernardis, P. \& Gentilucci, M. (2006). Speech and gesture share the same communication system. Neuropsychologia, 44(2), 178-190.

Boyatzis, C. J. \& Watson, M. W. (1993). Preschool children's 
symbolic representation of objects throught gestures. Child Development, 64, 729-735.

Camaioni, L., Aureli, T., Bellagamba, F. \& Fogel, A. (2003). A longitudinal examination of the transition to symbolic communication in the second year of life. Infant and Child Development, 12, 1-26.

Capirci, O., Iverson, J. M., Pizzuto, E. \& Volterra, V. (1996). Gestures and words during the transition to two word speech. Journal of Child Language, 23, 645-673.

Capone, N. \& McGregor, K. (2004). Gesture development: A review for clinical and research practices. Journal of Speech, Language and Hearing Research, 47, 173-186.

Crais, E., Day, D. \& Cox, C. (2004). The intersection of the development of gestures and intentionality. Journal of Speech, Language and Hearing Research, 47(3), 678-694.

Daniels, M. (2001). Dancing with words: Signing for hearing children's literacy. Wesport, CT: Bergin \& Garvey.

Folven, R. J. \& Bonvillian, J. D. (1991). The transition from nonreferential to referential language in children acquiring American Sign Language. Developmental Psychology, 27(5), 806-816.

García, J. (1999). Sign with your baby. Seattle, WA: Northlight Communications.

Goldin-Meadow, S. (2000). Beyond words: The importance of gesture to researchers and learners. Child Development, 71(1), 231-239.

Goldin-Meadow, S. (2002). Constructing communication by hand. Cognitive Development, 17, 1385-1405.

Goldin-Meadow, S. \& Morford, M. (1985). Gesture in early child language: Studies of deaf and hearing children. Merril-Palmer Quarterly, 31, 145-176.

Gómez, J. C., Sarriá, E. \& Tamarit, J. (1993). El estudio comparado de la comunicación temprana y la teoría de la mente: Ontogénesis, filogénesis y patología. Siglo Cero, 24(6), 47-62.

Goodwyn, S. (1986). Mother-infant interaction and infants'spontaneous symbolic signing. Disertación doctoral no publicada, University of California, Davis, Estados Unidos.

Goodwyn, S. \& Acredolo, L. (1993). Symbolic gesture versus word: Is there a modality advantage for onset of symbol use? Child Development, 64, 688-701.

Goodwyn, S. \& Acredolo, L. (1998). Encouraging symbolic gestures: Effects on the relationship between gesture and speech. En J. Iverson \& S. Goldin-Meadow (Eds.), The nature and functions of gestures in children 's communication (pp. 61-73). San Francisco: Jossey-Bass.

Goodwyn, S., Acredolo, L. \& Brown, C. (2000). Impact of symbolic gesturing on early language development. Journal of Nonverbal Behavior, 24, 81-103.

Iverson, J. M., Capirci, O. \& Caselli, C. (1994). From communication to language in two modalities. Cognitive Development, $9(1), 23-43$.

Iverson, J. M., Capirci, O., Longobardi, E. \& Caselli, C. (1999). Gesturing in mother-child interactions. Cognitive Development, 14(1), 57-75.

Iverson, J. M. \& Goldin-Meadow, S. (1997). What's communication got to do with it? Gesture in children blind from birth. Developmental Psychology, 33(3), 453-467.
Kelly, S. (2001). Broadening the units of analysis in communication: Speech and nonverbal behaviors in pragmatic comprehension. Journal of Child Language, 28(2), 325-349.

McEachern, D. \& Haynes, W. (2004). Gesture-speech combinations as a transition to multiword utterances. American Journal of Speech-Language Pathology, 13(3), 227-235.

Mehler, J. \& Dupoux, E. (1994). Nacer sabiendo. Madrid: Alianza.

Moore, B., Acredolo, L. \& Goodwyn, S. (2001, abril). Symbolic gesturing and joint attention: Partners in facilitating verbal development. Ponencia presentada en Biennial Meetings of Society for Research in Child Development, Minneapolis, Estados Unidos.

Namy, L., Acredolo, L. \& Goodwyn, S. (2000). Verbal labels and gestural routines in parental communication with young children. Journal of Nonverbal Behavior, 24(2), 63-79.

Namy, L. \& Waxman, S. (1998). Word and gestures: Infants' interpretations of different forms of symbolic reference. Child Development, 69(2), 295-308.

Nicoladis, E., Mayberry, R. \& Genesee, F. (1999). Gesture and early bilingual development. Developmental Psyhology, $35(2), 514-526$.

O'Reilly, A. (1995). Using representations: Comprehension and production of actions with imagined objects. Child Development, 66, 999-1010.

O’Reilly, A., Painter, K. \& Bornstein, M. (1997). Relations between language and symbolic gesture development in early childhood. Cognitive Development, 12(2), 185-197.

Piaget, J. (1965). El lenguaje y pensamiento del niño pequeño. Buenos Aires: Paidós.

Premack, D. \& Woodruff, G. (1978). Does the chimpanzee have a theory of mind? The Behavioral and Brain Sciences, 4, 515-526.

Rodrigo, M. J., González, A., Ato, M., Rodríguez, G. \& de Vega, M. (2006). Co-development of child-mother gestures over the second and the third years. Infant and Child Development, 15, 1-17.

Thal, D., \& Bates, E. (1988). Language and gesture in late talkers. Journal of Speech and Hearing Research, 31(1), 115-123.

Thal, D., Tobias, S. \& Morrison, D. (1991). Language and gesture in late talkers: A 1-year follow-up. Journal of Speech and Hearing Research, 34(3), 604-612.

Tomasello, M. \& Farrar, M. (1986). Joint attention in early language. Child Development, 57, 1454-1463.

Vallotton, C. (2005). Effects of symbolic gestures as a caregiving tool: Children's social and language development and mother's perceptions and behavior. Dissertation Abstracts International, 65(9), 4877B.

Vallotton, C. (en prensa). Signs of emotion: What can preverbal children "say" about internal states? Journal of Infant Mental Health.

Volterra, V., Caselli, M. C., Capirci, O. \& Pizzuto, E. (2005). Gesture and the emergence and development of language. En M. Tomasello \& D. Slobin (Eds.), Beyond nature-nurture: Essays in honor of Elizabeth Bates (pp. 3-39). Mahwah, NJ: Lawrence Erlbaum.

Fecha de recepción: noviembre de 2006.

Fecha de aceptación: julio de 2007. 